\title{
Simultaneous demonstration of acid phosphatase and glucose-6-phosphate dehydrogenase in mouse hepatocytes. A novel electron-microscopic dual staining enzyme-cytochemistry
}

\author{
S. Matsubara
}

Department of Obstetrics, Gynecology and Anatomy, Jichi Medical School, Minamikawachi-machi 3311-1, Kawachi-gun, Tochigi 329-0498, Japan

Key Words: acid phosphatase, glucose-6-phosphate dehydrogenase, hepatocyte, enzyme-cytochemistry, electron microscopy

\section{SUMMARY}

Acid phosphatase (ACPase) and glucose-6-phosphate dehydrogenase (G6PD) play important roles in cell biology/disease pathophysiology in various organs including the liver. The purpose of the present report is to introduce a new enzymecytochemical method to simultaneously demonstrate the subcellular localization of ACPase and G6PD within the same hepatocyte in the mouse liver. The ultrastructural localization of ACPase and G6PD were demonstrated, with concomitant use of the cerium method and the copper-ferrocyanide method, respectively. ACPase labelings were localized in the lysosomes, and G6PD labelings were visible in the cytoplasm and on the cytosolic side of the endoplasmic reticulum of the hepatocyte. This novel double staining procedure may be a useful histochemical tool for the study of liver functions in both physiological and pathological conditions.

\section{INTRODUCTION}

Acid phosphatase (ACPase; E.C. 3.1.3.2) and glucose-6-phosphate dehydrogenase (G6PD; E.C.1.1.-
1.49) are important enzymes in various cells, tissues and organs, including the liver parenchymal cells (hepatocytes). The lysosomal enzyme, ACPase, is discharged into phagosomes/phago-lysosomes, and it hydrolyses/digests undesirable substances phagocytosed within phagosomes/phago-lysosomes of hepatocytes, and thus defending the liver against external noxious stimuli (Gross et al., 1989). In various liver diseases, however, ACPase, spilling out through the weakened lysosomal membrane, may also attack the liver, leading to cell death/necrosis (Yasuda et al., 2000). Reactive oxygen species (ROS), produced within hepatocytes, may lead to the weakening or disruption of the lysosomal/plasma membranes (Yasuda et al., 2000).

G6PD is a key enzyme of the pentose phosphate pathway in carbohydrate metabolism and is contained abundantly in hepatocytes. This enzyme catalyzes the conversion of glucose-6-phosphate to 6phosphogluconate, with concomitant production of NADPH and ribose-5-phosphate. The former substance, NADPH, is used as essential substance/substrate for NADPH-dependent ROS producing oxidoreductase enzymes (such as NADPH oxidase and nitric oxide synthase) (Tian et al., 1999). G6PD, in 
one way affecting the redox potential within cells by supplying NADPH itself, and in another way regulating ROS producing enzyme activities, is reported to have a close association with various cell functions/activities including cell death/necrosis (Tian et al., 1999). The latter substance, ribose-5-phosphate, is used in DNA/RNA synthesis within cells. G6PD, therefore, plays important roles in liver biology. Since ACPase and G6PD function co-ordinatedly in hepatocytes, and since they may play essential roles in both liver biology and liver disease pathophysiology, it is worthy demonstrating these two enzymes simultaneously in the same hepatocyte at the subcellular level.

Recently, we developed a G6PD enzyme-cytochemistry method using copper ferrocyanide as the capturing agent (the copper-ferrocyanide method), which enabled us to determine the fine subcellular localization sites of G6PD within cells (Ishibashi et al., 1999). I performed the present study to simultaneously localize ACPase and G6PD in mouse hepatocytes, with concomitant use of the cerium method for ACPase (Robinson and Karnovsky 1983) and the copper-ferrocyanide method for G6PD (Ishibashi et al., 1999).

\section{MATERIALS AND METHODS}

Five male ICR adult mice (obtained from CLEA Japan, Tokyo, Japan) were used for this study. They were maintained on ordinary laboratory chow and water ad libitum with humane care. Livers were removed under pentobarbital anesthesia, cut into small strips less than $1 \mathrm{~mm}$ thick, and fixed in a $2.0 \%$ glutaraldehyde solution $(0.1 \mathrm{M}$ cacodylate buffer $(\mathrm{pH}$ 7.4) with $10 \%$ sucrose) for $30 \mathrm{~min}$ at $4^{\circ} \mathrm{C}$. After washing in a cacodylate buffer $(0.1 \mathrm{M}, \mathrm{pH} 7.4)$ for 3 $\mathrm{h}$, the samples were cut into $40 \mu \mathrm{m}$-sections with a freezing microtome. The sections were incubated in a reaction medium for G6PD first, followed by detection for ACPase. G6PD activity was detected using the copper-ferrocyanide method (Ishibashi et al., 1999). In brief, sections were incubated in a reaction medium for $60 \mathrm{~min}$ at $37^{\circ} \mathrm{C}$ in the dark with constant agitation. The reaction medium consisted of:/ $10.5 \mathrm{mM}$ G6P (disodium salt), $70 \mathrm{mM}$ phosphate buffer ( $\mathrm{pH} 7.2$ ), $1.3 \mathrm{mM}$ NADP, $10 \mathrm{mM}$ sodium citrate, $1.5 \mathrm{mM}$ copper sulfate, $0.5 \mathrm{mM}$ potassium ferricyanide, $1.0 \mathrm{mM}$ phenazine methosulfate, and $267 \mathrm{mM}$ sucrose. After washing for $15 \mathrm{~min}$ in a cacodylate buffer $(0.1 \mathrm{M}, \mathrm{pH} 7.4)$ with 3 buffer changes, the sections were incubated in the cerium medium for the detection of ACPase for $15 \mathrm{~min}$ at $37^{\circ} \mathrm{C}$. The cerium medium consisted of $1 \mathrm{mM}$ betaglycerophosphate, $100 \mathrm{mM}$ acetate buffer ( $\mathrm{pH}$ 5.0), $2.0 \mathrm{mM}$ cerium chloride and $267 \mathrm{mM}$ sucrose (Robinson and Karnovsky 1983; Matsubara et al., 2000). Sections were postfixed in $1.0 \%$ buffered osmium tetroxide for $60 \mathrm{~min}$ at $4^{\circ} \mathrm{C}$, dehydrated, and embedded in epoxy resin. Ultrathin sections were prepared, with or without counterstaining with uranyl acetate and lead citrate, and observed under a transmission electron microscope. To ensure the specific detection of G6PD activity, cytochemical control experiments were performed as follows (Ishibashi et al., 1999): (1) Sections were incubated in a medium (a) lacking substrate (G6P), (b) devoid of NADP, followed by incubation in a complete ACPase medium, and (2) sections were heated at $100^{\circ} \mathrm{C}$ for $10 \mathrm{~min}$, then incubated in the dual reaction medium. I further performed G6PD enzymecytochemistry (without ACPase staining) and ACPase enzymecytochemistry (without G6PD staining). The experimental conditions were the same as the double staining procedure cited above, except that the sections were not incubated in another reaction medium. Finally, to confirm the specific detection of ACPase activity detected by single ACPase staining, cytochemical control experiments were further performed as; (1) omission of the substrate (betaglycerophosphate) from the reaction medium, and (2) addition of ACPase-inhibitor (10 $\mathrm{mM} \mathrm{NaF})$ to the medium (Matsubara et al., 1987).

\section{RESULTS}

When mouse liver sections were incubated in ACPase medium (without G6PD staining), the precipitates were visible in the hepatocyte cytoplasmic vacuoles, which were round, ovoid, or elongated (Fig. A). The omission of the substrate (the substrate free control) and the addition of the ACPase-inhibitor (10 mM NaF) completely abolished the electron-dense precipitates within these vacuoles (data not shown). ACPase-positive structures were considered to be lysosomes. When sections were incubated in the reaction medium for G6PD (without ACPase staining), electron-dense 
precipitates indicating G6PD activity were visible in the cytoplasm and on the cytosolic side of the endoplasmic reticulum (ER) membranes (Fig. B). When liver tissues were incubated in the reaction medium for G6PD followed by ACPase medium (dual reaction method), dense deposits were visible in the lysosomes, cytoplasm and the cytosolic side of ER (Fig. C1-4); the former (lysosome) represented ACPase activity, and the latter two (cytoplasm and ER) showed G6PD activity. Distribution pattern and intensities did not differ among the mice studied (no individual differences). When the incubation order was reversed (ACPase first, then G6PD), the intensities of ACPase remained the same, but G6PD labelings became a little fainter (data not shown). When tissues were incubated in the reaction medium lacking the substrate for G6PD (G6P) or NADP, precipitates in the cytoplasm and on ER completely disappeared, but lysosomes still showed electron-dense precipitates (Fig. D). Heating the section abolished all the depositions (data not shown).

\section{DISCUSSION}

Enzymecytochemistry has been giving great contribution to clarifying the biology and pathophysiology of various organs including the liver. Simultaneous demonstration of two enzymes may further contribute to the research for cell biology/pathophysiology, only if the corresponding enzymes are biologically significant, and thus worthy of investigation. The present attention has been focused on the hepatocytes not only because both ACPase and G6PD play important roles in the liver biology and pathophysiology, but also because the hepatocytes, possessing marked activities of various enzymes including ACPase and G6PD, suit well for developing novel enzyme-cytochemical methods.

Electron-dense deposits were observed in the following three intra-cellular compartments; 1 ) in the lysosomes, 2) throughout the cytoplasm, and 3) on the cytosolic side of ER. The deposits in the former organelles represented ACPase activity, and the latter two indicated G6PD activity. Previous biochemical and histochemical studies indicated that ACPase is packed within lysosomes (Robinson and Karnovsky 1983; Matsubara et al., 2000). The cytoplasm and ER did not show cytochemically detectable ACPase (Robinson and Karnovsky 1983; Matsubara et al., 2000). The present cytochemical negative control experiments also confirmed the specific detection of ACPase within lysosomes. Thus, I conclude that precipitates in the lysosomes obtained in the present dual staining procedure represent ACPase. Regarding G6PD localization, previous biochemical (Ozols 1993), immuno-electron microscopic (Ninfali et al., 2000), and single G6PD enzyme-cytochemical (Ishibashi et al., 1999; Matsubara et al., 2001; Matsubara et al., 2002) studies indicated that G6PD resided both in the cytoplasm and in close association with ER in various cells: these findings coincide well with the present observations obtained by the dual staining method. The cytochemical negative control experiments also ensured the specific detection of G6PD obtained here. Thus, I conclude that the deposits in the cytoplasm and on ER represented true G6PD enzyme labelings. I therefore demonstrated enzyme-cytochemically detectable ACPase activity and G6PD activity at the same time within the same hepatocyte.

There have been some trials by other researchers for the simultaneous enzymecytochemical demonstration of two enzymes. For example, combined staining for peroxidase and ACPase was tried in mouse peritoneal macrophages (van Dort et al., 1989), in which the precipitates for both enzymes were clearly observed in the corresponding intracellular organelles when sections were first incubated in peroxidase medium, followed by a cerium medium for ACPase. When the sequences were reversed, peroxidase-related reaction products were not observed, indicating that the order in which the incubations were carried out may be of some importance. This may partly hold true for the present method, in which the order in reaction also affected the deposition intensities. Detection for G6PD first, and ACPase second, gave sufficient precipitates of both enzymes, without any enzyme diffusion or artifact deposition. When reversing the sequence, G6PD labelings became fainter. I do not know the reason for this, but one possible explanation is that the cerium contained in the reaction medium for ACPase may affect as yet undetected G6PD activity. Regardless of this, the present experimental conditions sufficed in simultaneous demonstration of ACPase and G6PD in mouse hepatocytes. 

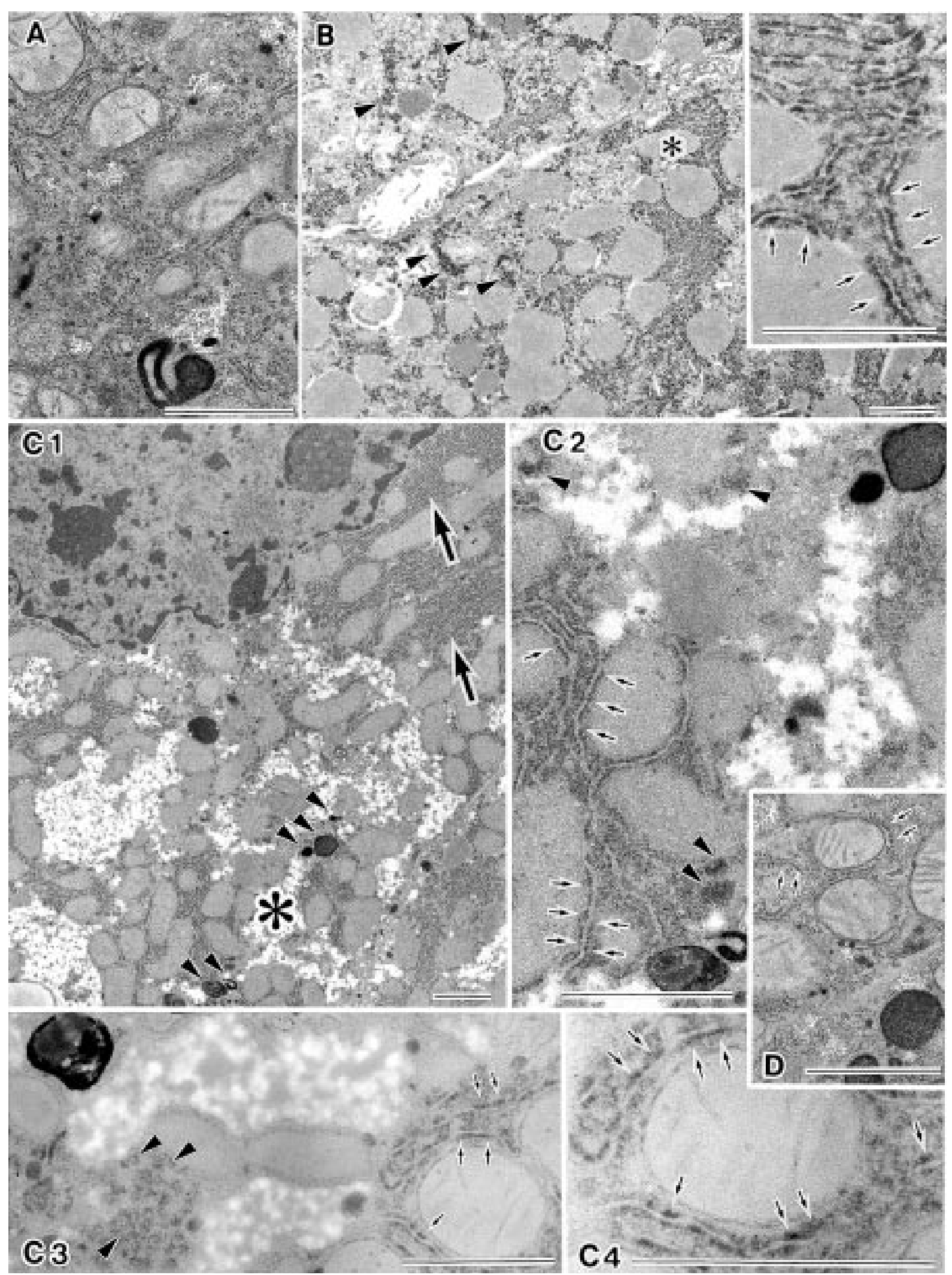
What is the biological/histochemical significance of the simultaneous demonstration of ACPase and G6PD in hepatocytes? I believe that this method may be a good tool for the elucidation of liver biology and liver disease pathophysiology. Both ACPase and G6PD play important roles in the liver function not only in the normal, but also the pathological condition. ACPase may be associated with albumin transport (Imai et al., 1994) and excretion of overloaded heavy metals from the liver (Gross 1989). It also plays important roles in the pathophysiology of various liver diseases including $\mathrm{CCl}_{4}$ - induced liver injury (Yasuda et al., 2000). Regarding G6PD, this enzyme regulates the pentose phosphate pathway of carbohydrate metabolism, playing essential roles in liver metabolic function, and has a close association with cell phagocytosis function (Corraliza et al., 1993). Furthermore, coordinated up-regulation of G6PD and NADPH-dependent ROS-generating enzyme (NADPH oxidase, nitric oxide synthase, etc.) activities has also been postulated as a key component of endotoxemia-induced liver damage (Spolarics and Navarro 1994). G6PD generates NADPH, a substrate for these ROS-generating enzymes. In various liver diseases, ROS, leading to lysosomal membrane instability, has a close association with liver cell dysfunction (Yasuda et al., 2000). Therefore, ACPase and G6PD must work together for the maintenance of the normal liver function, and they may affect each other in terms of liver physiology/pathophysiology. Although the present study itself did not produce direct evidence for these, the dual staining method introduced here has a potential to elucidate these important issues. Since these two enzymes play important roles in phagocytosis func- tion (Corraliza et al., 1993) and in ROS-ACPase associated hepatocyte necrosis (such as $\mathrm{CCl}_{4}$ induced liver injury) (Yasuda et al., 2000), dual staining method may be a good tool for elucidating the liver function/the liver disease pathophysiology, especially hepatocyte phagocytosis and hepatocyte necrosis.

Dual staining method may also be used for the study of other cells, tissues, and organs than the liver. Tissue macrophages may be good candidates, because ACPase and G6PD have a close association with phagocytosis function of phagocytes/macrophages as repeatedly cited. Our group, using single G6PD cytochemistry, already demonstrated that two types of tissue macrophages, ie. placental villous macrophages (Matsubara et al., 2001) and rat Kupffer cells (Matsubara et al., in submission), exhibited marked enzymecytochemically detectable G6PD activity. The dual staining for ACPase and G6PD introduced here enables us to determine two enzymes' subcellular localization within the same cell. The relationship between their intracellular distribution/intensities is also easily demonstrable on a cell-by-cell basis. This method may be a useful histochemical tool for the study of cell biology/disease pathophysiology.

\section{ACKNOWLEDGMENTS}

I would like to thank Dr. Toshihiro Takizawa, Dr. Takashi Yashiro (Department of Anatomy, Jichi Medical School), Dr. Ken-ichi Ido (Division of Gastroenterology, Jichi Medical School), and Dr.

Fig. 1 - Mouse hepatocyte enzyme activities (ACPase (A), G6PD (B) and simultaneous demonstration of ACPase and G6PD (C1-4) ) and its cytochemical control (D). Fig. (A), (B), (C1), (C2), and (D) are from specimens counterstained with uranyl acetate and lead citrate, and Fig. (C3) and (C4) are form specimens without counterstaining. Bar=1 $\mu \mathrm{m}$. (A) Electron-dense deposits indicating ACPase activities are visible in lysosomes. (B) G6PD labelings are observable in the cytoplasm (arrowheads) and in close association with ER. The latter are apparent in the inset at a higher magnification. Inset; The area marked by an asterisk in Fig. (B). Arrows indicate G6PD labelings on the cytosolic side of ER of the hepatocyte. (C1-4) Dual staining for ACPase and G6PD. (C1) ACPase labelings are present in the lysosomes (arrowheads). The richly ER-containing area marked by large arrows exhibits high-electron densities, which indicates G6PD labelings in ER, though their fine subcellular distributions are not confirmed at this low magnification. The area indicated by an asterisk will be shown at a higher magnification in Fig. (C2). (C2) G6PD labelings are visible in the cytoplasm (arrowheads) and in close association with ER (arrows). (C3) Unstained specimen. Electron-dense deposits indicating ACPase are visible in the lysosme. G6PD labelings are present in the cytoplasm (arrowheads) and in close association with ER (arrows). The right half area will be shown at a higher magnification in Fig. (C4). (C4) Unstained specimen. Arrows indicate G6PD labelings associated with ER. (D) Cytochemical negative control. The sections were incubated in the G6PD medium devoid of NADP, followed by the incubation in the complete ACPase medium. Deposits are observed only in lysosomes. Counterstained ribosomes are observed on the rough ER membranes (arrows), but electron-densities and staining patterns clearly distinguish between ribosomes and G6PD labelings on ER [comparison between the deposits shown in the inset of Fig. (B), (C2), (C3), and (C4) vs (D)]. 
Ikuo Sato (Department of Obstetrics and Gynecology, Jichi Medical School) for their helpful comments for this study.

\section{REFERENCES}

Corraliza I.M., Campo M.L., Fuentes J.M., Campos-Portuguez S., and Soler G.: Parallel induction of nitric oxide and glucose-6-phosphate dehydrogenase in activated bone marrow derived macrophages. Biochem. Biophys. Res. Comm. 196, 342-347, 1993.

Gross J.B., Myers B.M., Kost L.J., Kuntz S.M., and LaRusso N.F.: Biliary copper excretion by hepatocyte lysosomes in the rat. Major excretory pathway in experimental copper overload. J. Clin. Invest. 83, 30-39, 1989.

Imai M., Ishii Y., Tada K., Kitagawa Y., Kashiwadani M., Kashima K., and Ibata Y.: Effects of colchicine on the synthesis and transport of albumin: a combined electron cytochemical and autoradiographic study. J. Gastroenterol. Hepatol. 9, 257-263, 1994

Ishibashi T., Takizawa T., Iwasaki H., Saito T., Matsubara S., Nakazawa E., and Kanazawa K.: Glucose-6-phosphate dehydrogenase cytochemistry using a copper ferrocyanide and its application to rapidly frozen cells. Histochem. Cell Biol. 112, 221-232, 1999

Matsubara S., Tamada T., and Saito T.: Ultracytochemical localization of alkaline phosphatase and acid phosphatase activities in the human term placenta. Acta Histochem. Cytochem. 20, 283-294, 1987.

Matsubara S., Takizawa T., and Sato I.: Enzyme histochemistry on human placental trophoblasts: The effect of fixation delay on enzyme activity. Histochem. Cell Biol. 113, 287-292, 2000.

Matsubara S., Takayama T., Iwasaki R., Komatsu N., Matsubara D., Takizawa T. and Sato I.: Enzyme-cytochemically detectable glucose-6-phosphate dehydrogenase in human villous macrophages (Hofbauer cells). Placenta 22, 882-885, 2001.

Matsubara S., Kato T., Oshikawa K., Yamada T., Takayama T., Koike T., Watanabe T., Izumi A., and Sato I.: Glucose-6-phosphate dehydrogenase in rat lung alveolar epithelial cells: An ultrastructural enzyme-cytochemical study. Eur. J. Histochem. 46, 243-249, 2002.

Ninfali P., Biagiotti E., Guidi L., Malatesta M., Gazzanelli G., and Grande P.D.: Cytochemical and immunocytochemical methods for electron microscopic detection of glucose-6phosphate dehydrogenase in brain areas. Brain Res. Protoc. 5, 115-120, 2000.

Ozols J.: Isolation and the complete amino acid sequence of luminal endoplasmic reticulum glucose-6-phosphate dehydrogenase. Proc. Natl. Acad. Sci. USA. 90, 5302-5306, 1993.

Robinson J.M., and Karnovsky M.J.: Ultrastructural localization of some phosphatases with cerium. J. Histochem. Cytochem. 31, 1197-1208, 1983
Spolarics Z. and Navarro L.: Endotoxin stimulates the expression of glucose-6-phosphate dehydrogenase in Kupffer and hepatic endothelial cells. J . Leukocyte Biol. 56, 453-457, 1994.

Tian W.N., Braunstein .LD., Apse K., Pang J., Rose M., Tian X., and Stanton R.C.: Importance of glucose-6-phosphate dehydrogenase activity in cell death. Am. J. Physiol. 276, C1121-1131, 1999.

van Dort J.B., Ketelaars G.A.M., Daems W.Th., and de Bruijin W.C.: Ultrastructural electron probe X-ray microanalytical reaction product identification of three enzymes in the same mouse resident peritoneal macrophage. Histochemistry 92 , 243-253, 1989.

Yasuda M., Okabe T., Itoh J., Takekoshi S., Hasegawa H., Nagata H., Osamura R.Y. and Watanabe K.: Differentiation of necrotic cell death with or without lysosomal activation: Application of acute liver injury models by carbon tetrachloride (CCL4) and dimethylnitrosamine (DMN). J. Histochem. Cytochem. 48, 1331-1340, 2000. 